\title{
Pharmacokinetics and Central Accumulation of Delta-9-Tetrahydrocannabinol (THC) and its Bioactive Metabolites Are Influenced by Route of Administration and Sex
}

\author{
Samantha L. Baglot ${ }^{1}$, Catherine Hume ${ }^{1}$, Gavin N. Petrie ${ }^{1}$, Robert J. Aukema ${ }^{1}$, Savannah
} H.M. Lightfoot ${ }^{1}$, Laine M. Grace ${ }^{1}$, Ruokun Zhou ${ }^{2}$, Linda Parker ${ }^{3}$, Jong M. Rho ${ }^{4}$, Stephanie L. Borgland $^{1}$, Ryan J. McLaughlin ${ }^{5}$, Laurent Brechenmacher ${ }^{2}$ and Matthew N. Hill ${ }^{1 *}$

\footnotetext{
${ }^{1}$ Department of Cell Biology and Anatomy, Hotchkiss Brain Institute, University of Calgary, Canada ${ }^{2}$ Southern Alberta Mass Spectrometry (SAMS) Facility, University of Calgary, Canada

${ }^{3}$ Department of Psychology and Collaborative Neuroscience Program, University of Guelph, Guelph, Canada

${ }^{4}$ Departments of Neurosciences and Pediatrics, University of California San Diego, and Rady Children's Hospital San Diego

${ }^{5}$ Department of Integrative Physiology and Neuroscience, Washington State University, Pullman, WA, USA
}

*Corresponding author:

Matthew Hill, $\mathrm{PhD}$

3330 Hospital Drive NW

Calgary, AB Canada T2N4N1

mnhill@ucalgary.ca 


\section{Highlights}

- Body temperature as well as blood and brain levels of THC and metabolites differ based on administration route

- THC inhalation results in immediate hypothermia, whereas THC injection results in delayed hypothermia

- THC inhalation results in higher initial brain THC levels than injection

- THC injection results in higher blood \& brain 11-OH-THC levels than inhalation

- Translational cannabis work should strongly consider using inhalation over injection 


\begin{abstract}
Up to a third of North Americans over 16 years old report using cannabis in the prior month, most commonly through inhalation. Animal models that reflect human cannabis consumption are critical to study its impacts on brain and behaviour. Nevertheless, most animal studies to date examine effects of cannabis through injection of delta-9-tetrahydrocannabinol (THC; primary psychoactive component of cannabis). THC injections produce markedly different physiological and behavioural effects than inhalation, likely due to distinctive pharmacokinetics of each administration route. The current study directly examined if administration route (injection versus inhalation), with dosing being matched on peak THC blood levels, alters the metabolism of THC, and the central accumulation of THC and its metabolites over time. Adult male and female Sprague-Dawley rats received either a single intraperitoneal injection of THC $(2.5 \mathrm{mg} / \mathrm{kg})$ or a single $(15 \mathrm{~min})$ session of inhaled exposure to THC distillate $(100 \mathrm{mg} / \mathrm{mL})$ vapour. Blood and brains were collected at 15, 30, 60, 90 and 240 minutes post-exposure for analysis of THC and metabolites through mass spectrometry-liquid chromatography. Inhalation results in immediate hypothermia, whereas injection results in delayed hypothermia. Despite achieving comparable peak concentrations of blood THC in both groups, our results indicate higher initial brain THC concentration following inhalation, whereas injection resulted in dramatically higher 11-OH-THC concentrations, a potent THC metabolite, in blood and brain that increased over time. Our results provide evidence that $\mathrm{THC}$ and its metabolites exhibit different pharmacokinetic profiles following inhalation versus injection, which could have significant impacts for data interpretation and generalizability. Accordingly, we suggest that translational work in the realm of THC and cannabis strongly consider using inhalation models over those that employ injection.
\end{abstract}

\title{
Keywords: THC; cannabis; rat; translational; vapour; injection
}




\section{1. Introduction}

With recreational use of cannabis recently becoming legal in Canada and in some states across

3 the U.S., as well as medicinal use being legal in many other countries, there is a growing need to better

4 understand the effects of cannabis on the brain and behaviour. Up to a third of North Americans over

516 years of age report using cannabis in the prior month (Goodman et al., 2020), most commonly

6 through pulmonary (i.e. inhalation) administration (Cuttler et al., 2016). Animal models provide an

7 extremely valuable approach to studying the effects of cannabis, enabling control over composition,

8 dose, and timing of exposure. Nevertheless, the majority of animal studies examine the effects of

9 cannabis through parenteral (i.e. intraperitoneal [IP]) injections of delta-9-tetrahydrocannabinol (THC;

10 the primary psychoactive component of cannabis) or cannabinoid receptor 1 (CB1R) agonists, which

11 does not reflect a common route of human cannabis consumption nor reproduce the same physiological

12 or behavioural effects of inhalation administration.

13 Inhalation of THC produces a much more rapid onset and offset of hypothermia, increases

14 feeding behaviour, decreases locomotion, and fails to induce cross-sensitization to morphine in rats

15 when compared directly to injected THC (Manwell et al., 2014; Nguyen et al., 2016). Further, while

16 THC injections result in conditioned avoidance, inhalation produces a place preference (Manwell et al.,

17 2014) and has reinforcing properties (as demonstrated by robust self-administration) in rats (Freels et

18 al., 2020). The effects of THC inhalation on temperature, appetite, and locomotion in rodents are also

19 typically shown in humans after exposure to cannabis smoke (Ashton, 2001; Kirkham and Williams,

20 2005; Sharma et al., 2012). The physiological and behavioural differences between inhalation and

21 injections are likely due to the distinctive pharmacokinetic (PK) differences of each route of

22 administration. 

through the liver to undergo metabolism before reaching other organs, such as the brain (Lukas et al., 1971; Turner et al., 2011). Alternatively, inhalation provides rapid delivery of compounds into the blood stream, bypassing initial metabolism by the liver, and resulting in more immediate uptake by highly perfused tissues, such as the brain (Huestis, 2007, 2005; Turner et al., 2011). The amount and duration of compound absorption also differs, with injections delivering a single bolus versus inhalation delivering an ongoing infusion. As such, the PK profile of THC, specifically plasma THC concentrations, between injections and inhalation differ in timing, magnitude, and duration.

The most common form of human cannabis administration - inhalation (smoking or vaporization) - produces peak plasma THC concentrations 10-15 min after initial administration (Hartman et al., 2015; Huestis et al., 1992; Huestis and Cone, 2004; Newmeyer et al., 2016; Schwope et al., 2011; Spindle et al., 2019, 2018) with relatively rapid clearance of THC from plasma. In fact, plasma THC concentrations are only $15-20 \%$ of peak at 30 min following cannabis use, $8-10 \%$ at 60 min, and 2-3\% at 180 min (Abrams et al., 2007; Newmeyer et al., 2016). However, as a result of individual differences in the number, duration, and spacing of puffs, as well as inhalation volume and hold time, the exact concentration of peak plasma THC in human studies is extremely variable. Peak plasma concentrations of THC range from $60-200 \mathrm{ng} / \mathrm{mL}$ following inhalation of cannabis flower (Abrams et al., 2007; Bidwell et al., 2020; Hartman et al., 2015; Huestis et al., 1992; Huestis and Cone, 2004; Newmeyer et al., 2016; Schwope et al., 2011), making animal models that can control dose and timing extremely valuable.

Rodent studies utilizing THC injections allow for control over both dose and timing; however, peak plasma THC concentrations are found at a slightly later timepoint following administration than with respect to inhalation (Javadi-Paydar et al., 2017; Nguyen et al., 2016; Taffe et al., 2020; Torrens et 
al., 2020). Further, clearance of THC from plasma following IP injections is much slower, with concentrations still roughly $65 \%$ of peak at $60 \mathrm{~min}$ and $50 \%$ at $120 \mathrm{~min}$ (Torrens et al., 2020). Rodent studies employing IP injections also utilize a wide range of dosages ( 3 to $20 \mathrm{mg} / \mathrm{kg}$ ) producing an extensive span of peak plasma THC concentrations from 40-200 ng/mL (Javadi-Paydar et al., 2017; Nguyen et al., 2016; Taffe et al., 2020; Torrens et al., 2020), suggesting that they are comparable to the range seen in humans following cannabis use. Interestingly, brain THC concentrations following IP administration increase over time, peaking at 60-120 min following initial administration (Torrens et al., 2020).

Animal models utilizing vapour delivery of THC or whole cannabis extract have recently been validated (Javadi-Paydar et al., 2017; Manwell et al., 2014; Nguyen et al., 2016) and are able to control for dose and timing of exposure while also employing the most common route of cannabis consumption in humans. Several rodent studies have found plasma THC concentrations of 100-200 ng/mL following $30 \mathrm{~min}$ of exposure to $100-200 \mathrm{mg} / \mathrm{mL}$ of THC vapour (Javadi-Paydar et al., 2017; Nguyen et al., 2020, 2016; Taffe et al., 2020). Similar to human inhalation, plasma THC concentrations peak at around 15 min (Hložek et al., 2017) with relatively rapid plasma clearance as suggested by concentrations of $\sim 30 \%$ of peak at $60 \mathrm{~min}$ and 8-10\% at $120 \mathrm{~min}$ (Javadi-Paydar et al., 2017; Nguyen et al., 2016; Taffe et al., 2020). Finally, opposite to injection, brain THC concentrations following inhalation peak at 15 min following initial administration and decrease over time (Hložek et al., 2017). In preclinical studies, dosing of THC is typically determined by whether it produces blood THC levels in the desired range seen in humans following cannabis consumption. However, whether route of administration influences how much THC, or its metabolites, accumulates in the brain and activates central cannabinoid CB1 receptors is not understood. As such, it is not clear if injections of THC that produce similar blood THC levels as those seen following inhalation are indeed comparable in the 
context of how much impact they have on activation of the central cannabinoid system. Consideration

of the metabolism of THC is also incredibly important in this context and is often not measured in most

analyses even though the metabolites of THC are highly bioactive, undoubtedly influenced by route of administration, and known to be significantly impacted by sex (Javadi-Paydar et al., 2017; Nguyen et al., 2020; Ruiz et al., 2021; Wiley et al., 2014). In the liver, THC is hydroxylated by cytochrome P450 enzymes into 11-hydroxy-THC (11-OH-THC), which is subsequently oxidized by the same group of enzymes to create 11-Nor-9-carboxy-THC (THC-COOH) and is excreted in urine (Huestis, 2005). The concentrations of THC metabolites are very important factors to consider when examining the impacts of THC administration, as 11-OH-THC is also psychoactive, is at least equipotent if not more potent than THC and diffuses more readily into the brain than THC (Grotenhermen, 2003; Lemberger et al., 1972; Perez-Reyes et al., 1972). Thus, differences in the generation and central accumulation of 11$\mathrm{OH}-\mathrm{THC}$ are not trivial and can have a robust impact on the outcome of studies given its ability to be as efficacious, if not more, as THC in activating $\mathrm{CB} 1$ receptors. $\mathrm{THC}-\mathrm{COOH}$ is detectable for weeks, lacks any known psychoactivity, yet may possess anti-inflammatory and analgesic effects (Burstein, 1999; Grotenhermen, 2003).

In our attempts to develop more translationally relevant models of THC and cannabis administration, we examined if there were PK differences in the metabolism and accumulation of THC between inhaled and injected routes of administration that could help ascertain if these approaches are interchangeable or if there are differences that need to be considered when interpreting animal research data through a translational lens. To this extent, we utilized inhalation and injection paradigms, in both male and female rats, that produced comparable peak plasma THC concentrations to see if these different routes of administration resulted in differential PK or central accumulation of THC and its 
own analytical approach using mass spectrometry-liquid chromatography, which allowed us to quantify

93 these molecules in both plasma and brain tissue.

\section{Methods}

\subsection{Animals and housing}

Adult male and female Sprague-Dawley rats were obtained from Charles Rivers Laboratories (St.

Constant, QC, Canada). Rats were pair-housed with ad libitum access to water and standard laboratory

chow and acclimated to a standard colony room (12 h light-dark cycle; constant temperature of $21 \pm$

$1^{\circ} \mathrm{C}$ ). Following $\sim 1$ week of acclimation rats were split into two administration groups (injection

101

[parenteral] and inhaled [pulmonary]) and each group was further sub-divided according to five

timepoints $(15,30,60,90$, and $240 \mathrm{~min})(\mathrm{n}=5-6$ per group). All animal procedures were performed in accordance with the Canadian Council on Animal Care (CCAC) guidelines and were approved by the University of Calgary Animal Care Committee.

\subsection{Injections of THC}

Dosing for both injection and inhalation studies was based on pilot work establishing doses that produced roughly comparable blood THC levels in the range of 60-100 ng/mL. Rats received a single injection of THC intraperitoneally (dose of $2.5 \mathrm{mg} / \mathrm{kg}$ in a volume of $2 \mathrm{ml} / \mathrm{kg}$ ). THC (100 mg/mL in

\section{$110100 \%$ EtOH from Toronto Research Chemicals) was stored at $-20^{\circ} \mathrm{C}$ until dissolved into a 1:1:8}

111 solution of dimethylsulfoxide (DMSO), Tween 80, and 0.9\% saline respectively. Following injections

112 rats were euthanized via decapitation at five different timepoints $(15,30,60,90$ and $240 \mathrm{~min}$; referred

113 to as INJ 15, INJ 30, INJ 60, INJ 90 and INJ 240 hereafter); trunk blood was collected and brains were

114 extracted for hippocampus dissection. The hippocampus was chosen as the brain structure for analysis 
as it is an important site for many of the cognitive and emotional effects of cannabinoids, has a high

116 density of cannabinoid receptors and is a brain structure whose isolation and dissection is consistent

117 and straightforward. Blood samples were collected in EDTA tubes and stored on ice until centrifuged at

$11810,000 \mathrm{rpm}$ for 10 minutes at $4^{\circ} \mathrm{C}$. Plasma was collected and stored at $-80^{\circ} \mathrm{C}$ until analysis. Dissected

119 hippocampi were immediately frozen on dry ice and then stored at $-80^{\circ} \mathrm{C}$ until analysis.

120

\subsection{Passive inhaled delivery of THC}

122

Rats received a single (15 $\mathrm{min})$ session of inhaled exposure to a cannabis-derived THC-distillate

123 (100 mg/mL) via a validated (Freels et al., 2020; Javadi-Paydar et al., 2017; Nguyen et al., 2016)

124 vapour inhalation system (La Jolla Alcohol Research Inc., CA, USA). THC distillate (95\% THC from

125 Aphria Inc., ON, CND) was stored at room temperature until diluted to a concentration of $100 \mathrm{mg} / \mathrm{mL}$

126 THC in polyethylene glycol (PEG-400). The vapour inhalation system uses machinery similar to

127 electronic cigarettes to deliver distinct "puffs" of cannabis vapour within airtight chambers. Chamber

128 airflow is controlled by a vacuum compressor (i.e. a "pull" system) that draws room ambient air

129 through an intake valve at a constant rate of $1 \mathrm{~L}$ per minute. At set intervals (as controlled by MedPC

130 IV software [Med Associates, ST. Albans, VT, USA]) THC distillate is vaporized (utilizing a SMOK

131 TFV8 X-baby atomizer [Shenzhen IVPS Technology Co., Shenzhen, China]) combining with the

132 constant ambient air flow for delivery into the chamber. Air (and vapour) are evacuated through the

133 back of the chamber via the vacuum compressor, filtered and ventilated out of the building (see Figure

1341 for illustrated depiction of the vapour delivery system). In this study, THC vapor was delivered

135 through a 10-second "puff” every 2 minutes for 15 minutes. In accordance with the injected animals,

136 following the conclusion of the vapour session rats were euthanized via decapitation at five different 
137 timepoints (15 [immediate], 30, 60, 90 and $240 \mathrm{~min}$, referred to as INH 15, INH 30, INH 60, INH 90

138 and INH 240 hereafter). Trunk blood and brains were collected and stored as previously described.

139

\subsection{Body temperature}

Body temperature was taken via a rectal thermometer immediately prior to euthanasia for all rats at the 30, 60, 90, and 240 min timepoints. As peak THC and metabolite levels were imperative to measure immediately following inhalation, blood and brain collection were prioritized at the $15 \mathrm{~min}$ timepoint. Further, hypothermic onset following THC exposure has been shown to occur at 30 min following inhalation (Manwell et al., 2014). Body temperature following THC administration was compared to control animals. Control animals were exposed to either vehicle vapour (PEG-400 alone)

147 for $15 \mathrm{~min}$ or to vehicle injection (1:1:8 DMSO, Tween 80 , and $0.9 \%$ saline) and their body

148 temperature was taken at 30, 60, 90 and 240 min post administration.

\subsection{Tandem mass spectrometry (LC-MS/MS)}

2.5.1 Standard solutions and reagents: Both standard and deuterated internal standard (IS) stock solutions were purchased from Cerilliant (Round Rock, TX, USA). The standard solutions, including $\Delta^{9}$-tetrahydrocannabinol (THC), 11-hydroxy-THC (11-OH-THC) and 11-nor-9-carboxy-THC (THC-

$154 \mathrm{COOH})$ were dissolved in acetonitrile at a concentration of $1.0 \mathrm{mg} / \mathrm{mL}$. The IS stock solutions 155 including THC-d3 and THC-COOH-d3 were dissolved in acetonitrile at $0.1 \mathrm{mg} / \mathrm{mL}$. LC/MS grade acetonitrile, water and formic acid were purchased from Thermo Fisher Scientific (Edmonton, Canada). All compounds and their serial dilutions were stored at $-80{ }^{\circ} \mathrm{C}$ freezer until use. 
2.5.2 Calibration curves: The stock solutions of each standard were mixed and diluted in 50\% methanol/water to produce a set of standards ranging from $0.1 \mathrm{ng} / \mathrm{mL}$ to $100 \mathrm{ng} / \mathrm{mL}(0.1,0.25,0.5,1$, $2.5,5,10,25,50,100)$. Internal standard (IS; d3 analytes) solution contains each compound at 10

$162 \mathrm{ng} / \mathrm{mL}$ and was prepared in 50\% methanol/water. Solutions used to establish calibration curves were 163 prepared by mixing $20 \mu \mathrm{L}$ of each standard solution and $20 \mu \mathrm{L}$ of IS solution. The calibrators were analyzed in triplicate and the resulting calibration curves were fit by line of regression using a weight of $1 / \mathrm{x}^{2} . \mathrm{R}^{2}$ of each calibration curve was at least 0.999 . Lower limit of quantitation (LLOQ) of each analyte was determined to be at $0.1 \mathrm{ng} / \mathrm{mL}$. prepared to receive plasma and brain samples. Each plasma sample was thawed at room temperature and $500 \mu \mathrm{L}$ was directly pipetted into the prepared tubes. Each brain tissue sample was weighed and

171 the frozen piece placed into the prepared glass tubes for manual homogenization with a glass rod until

172 resembling sand. All samples were then sonicated in an ice bath for $30 \mathrm{~min}$ before being stored overnight at $-20^{\circ} \mathrm{C}$ to precipitate proteins. The next day samples were centrifuged at $1800 \mathrm{rpm}$ at $4^{\circ} \mathrm{C}$

174 for 3-4 min to remove particulates and the supernatant from each sample was transferred to a new glass

175 tube. Tubes were then placed under nitrogen gas to evaporate. Following evaporation, the tube

176 sidewalls were washed with $250 \mu \mathrm{L}$ acetonitrile in order to recollect any adhering lipids and then again

177 placed under nitrogen gas to evaporate. Following complete evaporation, the samples were re-

178 suspended in $100 \mu \mathrm{L}$ of 1:1 methanol and deionized water. Resuspended samples went through two

179 rounds of centrifugation $\left(15000 \mathrm{rpm}\right.$ at $4^{\circ} \mathrm{C}$ for $\left.20 \mathrm{~min}\right)$ to remove particulates and the supernatant

180 transferred to a glass vial with a glass insert. Samples were then stored at $-80^{\circ} \mathrm{C}$ until analysis by LC-

181 MS / Multiple Reaction Monitoring (MRM). 
LC200 coupled to an AB Sciex QTRAP 5500 mass spectrometry (AB Sciex, Ontario, Canada) at the

system consisted of a CTC refrigerated autosampler (held at $10^{\circ} \mathrm{C}$ ), a six-port sample injection valve

carried out on an Eksigent Halo C18 column $(2.7 \mu \mathrm{m}, 0.5 \times 50 \mathrm{~mm}, 90 \AA \AA$, AB Sciex $)$ at $40{ }^{\circ} \mathrm{C}$. The

mobile phase A was composed of $0.1 \%$ formic acid in water and the mobile phase $\mathrm{B}$ of $0.1 \%$ formic

acid in acetonitrile. The analytes $(2 \mu 1$ injection) were eluted at $30 \mu 1 / \mathrm{min}$ using a gradient from 25 to

95\% B in $2.5 \mathrm{~min}$. The column was then cleaned and regenerated using the following program: $95 \% \mathrm{~B}$

for $2 \mathrm{~min}, 95$ to $25 \% \mathrm{~B}$ in $0.2 \mathrm{~min}$ and $25 \% \mathrm{~B}$ for $2.3 \mathrm{~min}$. Before each injection, the column was

equilibrated at initial LC condition for 1 min. Carryover was checked by injection of a blank in

between samples. The data were acquired in positive electrospray ionization (ESI) and MRM mode.

MRM transitions and collision energies (CE) of the different compounds are listed in Table 1. Each

compound was acquired with two transitions. The first one was used to quantify the compound and the

second one to discriminate isomers when necessary. Ion spray voltage was set at $5500 \mathrm{~V}$. Nebulizer

198 gas (GS 1), auxiliary gas (GS 2), curtain gas (CUR) were set at 30, 30, 35 (arbitrary units),

respectively. Collision gas was set to Medium. Declustering potential (DP), entrance potential (EP)

and cell exit potential (CXP) were set at 80,7 and $14 \mathrm{~V}$, respectively. LC-MS/MRM data were

extracted ion chromatogram (XIC; peak area) normalized by the peak area of its corresponding IS. 


\subsection{Statistical analysis}

All data are expressed as mean \pm SEM. Data were analyzed using IBM SPSS Statistics 26 and graphed using GraphPad Prism 8. Basal body temperature differed between males and females, so the two sexes were analyzed separately. Temperature data were analyzed by three-way ANOVA with drug group (THC and control), administration group (injection and inhalation), and timepoint (30, 60, 90, and $240 \mathrm{~min}$ ) as between-subjects' factors. Analyte data were analyzed by three-way ANOVA with sex (male and female), administration group (THC INH or INJ), and timepoint (15, 30, 60, 90 and $240 \mathrm{~min}$ ) as between-subjects' factors. Post-hoc comparisons used Bonferroni post hoc tests and differences were considered significant at $\mathrm{p} \leq 0.05$.

\section{Results}

\subsection{Body Temperature}

Body temperature measures were compared to controls and analyzed separately by sex (female $218>$ male, main effect of $\operatorname{sex}\left[\mathrm{F}_{(1,136)}=23.219\right.$ at $\left.\left.\mathrm{p}<0.00001\right]\right)$. THC exposure resulted in hypothermia in male rats differentially depending on administration group (interaction effect of group and timepoint: $F(9,52)=5.831$ at $p<0.0001$, see Figure 2A). In particular, THC INH resulted in immediate hypothermia at 30 and $60 \mathrm{~min}(30 \mathrm{~min}: \mathrm{THC}$ INH $<\mathrm{CON}$ INH, CON INJ, and THC INJ at $\mathrm{p}<0.05 ; 60 \mathrm{~min}: \mathrm{THC}$ $\mathrm{INH}<\mathrm{CON}$ INJ and THC INJ at $\mathrm{p}<0.05)$, whereas THC INJ resulted in delayed hypothermia at 90 and 240 min (90 min: THC INJ $<$ CON INJ and THC INH at $\mathrm{p}<0.05 ; 240 \mathrm{~min}: \mathrm{THC}$ INJ $<$ CON INJ, at 30 min compared to 90 and $240 \min (\mathrm{p}<0.005)$, as well as remained lower at 60 min compared to 90 $\min (\mathrm{p}=0.014)$. In contrast, THC INJ resulted in lower body temperature at 90 min compared to 30 and $60 \min (\mathrm{p}<0.05)$, as well as remained lower at 240 min than all other timepoints $(\mathrm{p}<0.002)$. 
Females had an extremely similar pattern where THC exposure resulted in hypothermia in

229 female rats differentially depending on administration group (interaction effect of group and time:

$230 \mathrm{~F}(9,56)=7.097$ at $\mathrm{p}<0.00001$, see Figure $2 \mathrm{~B})$. In particular, THC INH resulted in immediate hypothermia

231 at 30 and $60 \mathrm{~min}(30 \mathrm{~min}: \mathrm{THC}$ INH $<\mathrm{CON}$ INH, CON INJ, and THC INJ at $\mathrm{p}<0.001 ; 60 \mathrm{~min}$ : THC

$232 \mathrm{INH}<\mathrm{CON}$ INH, CON INJ and THC INJ at $\mathrm{p}<0.05)$, whereas THC INJ resulted in delayed

233 hypothermia at 90 and $240 \mathrm{~min}(90 \mathrm{~min}: \mathrm{THC}$ INJ $<$ CON INJ, CON INH and THC INH at p<0.002;

234240 min: THC INJ $<$ CON INJ and THC INH at $p<0.001)$. Along these lines, THC INH resulted in

235 lower body temperature at 30 min compared to all other timepoints $(\mathrm{p}<0.05)$. Oppositely, THC INJ

236 resulted in lower body temperature at 90 and $240 \mathrm{~min}$ compared to 30 and $60 \mathrm{~min}(\mathrm{p}<0.001)$.

239 Control values serve as assay controls and were undetectable. Analyte concentrations were 240 therefore compared across THC administration groups and sex. THC chromatogram illustrates THC 241 (black line) and THC-d3 (grey line) with an overlapping peak at 2.7 min (see Figure 3A).

242 In females, but not males, plasma THC concentrations were higher following INH than INJ 243 regardless of timepoint (interaction effect of sex and group: $F(1,94)=11.164$ at $p=0.0012$, post hoc 244 female INH $>$ female INJ at $\mathrm{p}=0.0002$, see Figure 3B). Following injection, but not inhalation, males 245 had higher plasma THC concentrations than females regardless of timepoint (male INJ $>$ female INJ at $246 \mathrm{p}=0.0002)$. Further, regardless of group, males and females differed in plasma THC concentrations 247 across timepoints (interaction effect of sex and timepoint: $\mathrm{F}(4,94)=4.107$ at $\mathrm{p}=0.004$, post hoc male $30>$ 248 all timepoints at $\mathrm{p}>0.003$, male $15>$ male 90 at $\mathrm{p}=0.042$, male $240<$ male $15 / 30 / 60$ at $\mathrm{p}<0.002$, female $24915>$ all timepoints at $p<0.02$, female $30>$ female $60 / 90 / 240$ at $p<0.02$ see Figure $3 B$ ). Males also had 250 higher plasma THC concentrations than females at 30 and $60 \mathrm{~min}$ (post hoc male $30>$ female 30 at 
$251 \mathrm{p}=0.0005$ and male $60>$ female 60 at $\mathrm{p}=0.031$ ). Finally, regardless of sex, plasma THC concentrations 252 were higher following inhalation than injection at $15 \mathrm{~min}$ but not different at any other timepoint 253 (interaction effect of group and timepoint: $\mathrm{F}(4,94)=5.301$ at $\mathrm{p}=0.004$, post hoc INH $15>\mathrm{INJ} 15$ at $254 \mathrm{p}=0.00002$, see Figure 3B). Plasma THC concentrations also differed by group across timepoint, such 255 that following inhalation, plasma THC was higher at 15-min than all other timepoints and higher at 30256 min than later timepoints (post hoc INH $15>$ all timepoint at $p<0.01$ and INH $30>$ INH $60 / 90 / 240$ at $257 \mathrm{p}<0.03$, see Figure 3B), whereas following injection, plasma THC was higher at 30-min compared to 258 all other timepoints and lower at 240-min compared to all other timepoints (post hoc INJ $30>$ all 259 timepoints at $\mathrm{p}<0.002$ and INJ $240<$ all timepoints at $\mathrm{p}<0.008$ ).

260 Brain THC concentrations were higher following INH than INJ at 15, 30 and 60 min regardless 261 of sex (interaction effect of group and timepoint: $F(4,95)=7.791$ at $p=0.00002$; post-hoc INH $15>$ INJ 15 262 at $\mathrm{p}<0.00001$, INH $30>$ INJ 30 at $\mathrm{p}=0.00004$, and INH $60>$ INJ 60 at $\mathrm{p}=0.003$, see Figure $3 \mathrm{C}$ ).

263 Further, brain THC concentrations were higher at 15, 30 and 60 min compared to 90 and 240 min 264 following inhalation (post-hoc INH $15>$ INH 90 and 240 at p<0.003; INH $30>$ INH 90 and 240 at $265 \mathrm{p}<0.001$; INH $60>$ INH 90 and 240 at $\mathrm{p}<0.01)$. Alternatively, brain THC concentrations were higher at $26690 \mathrm{~min}$ than 15 and $240 \mathrm{~min}$, and trending higher compared to $30 \mathrm{~min}$, following injection (post-hoc 267 INJ $90>$ INJ 15 at $\mathrm{p}=0.006 ;$ INJ $90>$ INJ 30 at $\mathrm{p}=0.07 ;$ INJ $90>$ INJ 240 at $\mathrm{p}=0.003$ ). Interestingly, 268 brain THC concentrations were higher in males than females, regardless of group or timepoint (main 269 effect of sex: $F(1,95)=9.482$ at $p=0.003$, see Figure $3 C$ ).

270

\subsection{1-OH-THC}


274 higher following INJ than INH at all timepoints regardless of sex (interaction effect of group and 275 timepoint: $\mathrm{F}(4,95)=4.412$ at $\mathrm{p}=0.003$; post-hoc INH $15<$ INJ 15 at $p=0.03$, INH $30<$ INJ 30 at p $<0.00001$, INH $60<$ INJ 60 at p $<0.00001$, INH $90<$ INJ 90 at $p<0.00001$, INH $240<$ INJ 240 at

$277 \mathrm{p}=0.037)$. Further, while plasma 11-OH-THC concentrations did not differ across timepoint following 278 inhalation, following injection plasma 11-OH-THC concentration were lower at 15 min compared to 279 30, 60, and $90 \mathrm{~min}$ (post-hoc INJ $15<$ INJ 30 at $\mathrm{p}=0.0002$, INJ $15<$ INJ 60 at $\mathrm{p}=0.038$, INJ $15<$ INJ 90 at $\mathrm{p}=0.032$ ), as well as lower at $240 \mathrm{~min}$ compared to 30, 60 and $90 \mathrm{~min}$ (post-hoc INJ $240<$ INJ 30 281 at $\mathrm{p}<0.00001$, INJ $240<$ INJ 60 at $\mathrm{p}=0.0005$, INJ $240<$ INJ 90 at $\mathrm{p}=0.0005$ ). Interestingly, plasma 11282 $\mathrm{OH}-\mathrm{THC}$ concentrations were higher in females than males, regardless of group or timepoint (main 283 effect of sex: $F(1,95)=4.613$ at $p=0.034$, see Figure 4B).

In both sexes, brain 11-OH-THC concentrations were higher following INJ than INH regardless 285 of timepoint (interaction effect of sex and group: $\mathrm{F}(1,95)=5.356$ at $\mathrm{p}=0.023$; post hoc male $\mathrm{INH}<\mathrm{INJ}$ at 286 $\mathrm{p}=0.0002$ and female INH $<$ INJ at $\mathrm{p}<0.00001$, see Figure 4C). Further, following injection, brain 11287 OH-THC concentration were higher in females than males (post hoc male INJ $<$ female INJ at $288 \mathrm{p}<0.00001)$. Interestingly, regardless of sex, brain 11-OH-THC concentrations were higher following 289 INJ than INH at 30, 60 and 90 min (interaction effect of group and timepoint: $F(4,95)=6.411$ at $290 \mathrm{p}=0.0001 ;$ post hoc INH $30<$ INJ 30 at $\mathrm{p}=0.0002$, INH $60<$ INJ 60 at $p<0.00001$, INH $90<$ INJ 90 at $291 \mathrm{p}<0.00001$, see Figure 4C). Further, while brain 11-OH-THC concentrations did not differ across 292 timepoint following inhalation, following injection brain 11-OH-THC concentration were lower at 15 293 min compared to 30, 60, and $90 \mathrm{~min}$ (post-hoc INJ $15<$ INJ 30 at $\mathrm{p}=0.0009$, INJ $15<$ INJ 60 at $294 \mathrm{p}<0.00001$, INJ $15<$ INJ 90 at $\mathrm{p}<0.00001$ ), as well as lower at $30 \mathrm{~min}$ compared to $60 \mathrm{~min}$ (post hoc 295 INJ $30<$ INJ 60 at $\mathrm{p}=0.023$ ) and at $240 \mathrm{~min}$ compared to 30, 60 and $90 \mathrm{~min}$ (post-hoc INJ $240<$ INJ 30 296 at $\mathrm{p}=0.001$, INJ $240<$ INJ 60 at $\mathrm{p}<0.00001$, INJ $240<$ INJ 90 at $\mathrm{p}<0.00001)$. 


\subsection{THC-COOH}

THC-COOH chromatogram (see Figure 5A) illustrates THC-COOH (black line) and THC-

COOH-d3 (grey line) with an overlapping peak at $2.0 \mathrm{~min}$. In both sexes, plasma THC-COOH

concentrations were higher following INJ than INH regardless of timepoint (interaction effect of sex

and group: $\mathrm{F}(1,93)=12.241$ at $\mathrm{p}=0.0007$; post hoc male $\mathrm{INH}<\mathrm{INJ}$ at $\mathrm{p}=0.00006$ and female $\mathrm{INH}<\mathrm{INJ}$

at $\mathrm{p}<0.00001$, see Figure 5B). Further, following injection, plasma THC-COOH concentration were

higher in females than males (post hoc male INJ $<$ female INJ at $\mathrm{p}<0.00001$ ). Interestingly, regardless

of sex, plasma THC-COOH concentrations were higher following INJ than INH at 30, 60, 90 and 240

min (interaction effect of group and timepoint: $F(4,93)=3.0$ at $p=0.022$; post hoc INH $30<$ INJ 30 at

$\mathrm{p}<0.00001$, INH $60<$ INJ 60 at $\mathrm{p}<0.00001$, INH $90<$ INJ 90 at $\mathrm{p}<0.00001$, INH $240<$ INJ 240 at

$\mathrm{p}=0.00003$, see Figure 5B). Further, while plasma THC-COOH concentrations did not differ across

timepoint following inhalation, following injection plasma THC-COOH concentration were lower at 15

min compared to all other timepoints (post-hoc INJ $15<$ all timepoints at $\mathrm{p}<0.003$ ).

Brain THC-COOH concentrations were higher in females than male regardless of group or timepoint (main effect of sex: $\mathrm{F}(1,95)=15.442$ at $\mathrm{p}=0.0002$, see Figure 5C). Further, brain THC-COOH concentrations were higher following inhalation than injection regardless of sex or timepoint (main effect of group: $F(1,95)=56.656$ at $\mathrm{p}<0.00001$, see Figure 5C).

\section{Discussion}

Given the increased usage, demand, and potency of cannabis over the last few years (ElSohly et closely reflects human consumption is critical in order to study the impact on brain and behaviour. Nevertheless, most animal studies to date examine the effects of cannabis through IP injection of THC, 
which produces markedly different behavioural effects than inhalation (Manwell et al., 2014). In efforts

321 to make THC injection studies possess more face validity for translatability to humans, these previous

322 studies aimed to produce peak plasma THC concentrations that are comparable to concentrations in

323 human cannabis smokers. Utilizing 'comparable' dosages (based on their ability to produce blood THC

324 levels in the range produced in humans from cannabis) established in previous literature (Javadi-Paydar

325 et al., 2017; Nguyen et al., 2016) as well as pilot work in our laboratory, we sought to produce similar

326 peak plasma THC concentrations following inhalation and injection that are within the range typically

327 seen following cannabis consumption in humans (Abrams et al., 2007; Hartman et al., 2015; Huestis et

328 al., 1992; Huestis and Cone, 2004; Newmeyer et al., 2016; Schwope et al., 2011), to determine if route

329 of administration influenced metabolism or central accumulation of THC. Our data provide clear

330 evidence on the different physiological response and PK profiles of THC and its metabolites following

331 comparable dosing of inhaled versus injected THC, which could have significant impacts for data

332 interpretation and generalizability. Importantly, we found that inhalation led to immediate hypothermia

333 and an initial higher plasma and brain THC concentration, while injection led to delayed hypothermia,

334 dramatically higher 11-OH-THC concentrations in both plasma and brain and higher THC-COOH

335 concentration in the brain. Males in general had higher THC levels while females had higher metabolite

336 levels, supporting previous work that there are robust sex differences in the PK of THC.

Body Temperature: It is well established that THC exposure induces a hypothermic response in

339 both humans and animals (Huestis, 2007; Javadi-Paydar et al., 2017; Nguyen et al., 2020).

340 Hypothermia was found in both males and females following inhalation and injection of THC. In

341 accordance with previous literature (Javadi-Paydar et al., 2017; Nguyen et al., 2020), inhalation led to

342 immediate hypothermia with lower temperatures at 30 and 60 min following THC administration 
343 which normalized by 90 and $240 \mathrm{~min}$, whereas injection results in the opposite effect of delayed

344 hypothermia with lower temperatures at 90 and 240 min compared to 30 and 60 min following THC

345 administration. This hypothermic difference based on route of administration is not surprising given

346 that inhaled THC quickly enters the bloodstream and is taken up by the brain, whereas injected THC

347 undergoes metabolism by the liver before reaching the systemic circulation (Turner et al., 2011). Along

348 these lines, the onset of the hypothermic response seen in this study occurs in conjunction with peak

349 brain THC levels following both inhalation and injection, at 30 and 90 min respectively, indicating that

350 this is a good physiological proxy readout of central accumulation of cannabinoids. The impact of route

351 of administration on the temporal kinetics of hypothermia was not influenced by sex.

THC Concentrations in Plasma and Brain: Utilizing 'comparable' doses of THC inhalation and

injection, as determined by previous literature and our pilot work (Javadi-Paydar et al., 2017; Nguyen

et al., 2016), we show altered plasma THC levels across route of administration and timepoint.

356 Specifically, inhalation led to higher plasma THC concentrations than injection at 15 min, but the two

357 routes of administration did not differ at any other timepoint. This is not surprising as inhalation results

358 in much more rapid uptake into the bloodstream than injection. In fact, it is known that inhalation

359 produces peak plasma THC levels 10-15 min after initial administration in humans and rodents

360 (Hartman et al., 2015; Hložek et al., 2017; Huestis and Cone, 2004; Newmeyer et al., 2016; Schwope et

361 al., 2011), whereas peak levels are found at a slightly later timepoint following injection in rodents

362 (Javadi-Paydar et al., 2017; Nguyen et al., 2016; Taffe et al., 2020; Torrens et al., 2020). Along these

363 lines, plasma THC concentrations were highest following inhalation at 15 and 30 min compared to 60 ,

36490 and $240 \mathrm{~min}$. Alternatively, plasma THC concentrations were higher following injection at $30 \mathrm{~min}$

365 compared to all other timepoints. As anticipated, there was no difference between peak THC levels 
between the two routes of administration (peak THC following inhalation $73 \mathrm{ng} / \mathrm{mL}$ vs. peak THC

367 following injection $72 \mathrm{ng} / \mathrm{mL}$ ), and importantly these levels fall within the range found in human

368 studies (60-200 ng/mL) (Abrams et al., 2007; Hartman et al., 2015; Huestis et al., 1992; Huestis and

369 Cone, 2004; Newmeyer et al., 2016; Schwope et al., 2011).

370 Despite the similar peak plasma THC concentrations, inhalation led to higher brain THC

371 concentrations at 15,30 , and 60 min compared to injection. This is not surprising as cannabis

372 inhalation provides rapid delivery into the blood stream, bypasses initial liver metabolism, and results

373 in more immediate uptake by highly perfused tissues, such as the brain (Huestis, 2007, 2005; Turner et

374 al., 2011). Further, in accordance with previous literature showing earlier peak brain THC

375 concentrations following inhalation than injection (Hložek et al., 2017), we found brain THC levels

376 peaked at 15 and 30 min following inhalation, while peak brain THC levels did not occur until 90 min

377 following injection. Interestingly, the peak subjective "high" in humans following cannabis inhalation

378 is $\sim 30$ min following onset of administration (Grotenhermen, 2003), which corresponds to the higher

379 THC concentrations in the brain in this study following inhalation.

11-OH-THC Levels in Plasma and Brain: Overall, injection administration yielded significantly

382 higher plasma and brain concentrations of 11-OH-THC compared to inhalation. Specifically, both

383 plasma and brain concentrations of 11-OH-THC were relatively low ( $\sim 13$ and $\sim 22 \mathrm{ng} / \mathrm{mL}$ respectively)

384 following inhalation and did not differ across timepoints. Low concentrations of 11-OH-THC

385 following inhalation are common as concentrations are recirculated through the enterohepatic pathway

386 (i.e. liver to bile to small intestine back to liver) and quickly metabolized to THC-COOH

387 (Grotenhermen, 2003). Alternatively, plasma concentrations of 11-OH-THC were highest at 30, 60,

388 and 90 min compared to 15 and 240 min following injection, reaching average peak levels of $\sim 88$ 
$\mathrm{ng} / \mathrm{mL}$, which is about 8 times higher than inhalation levels. Further, brain concentrations of 11-OHTHC were also highest at 30, 60, and 90 min compared to 15 and 240 min following injection, reaching average peak levels of $\sim 98 \mathrm{ng} / \mathrm{g}$, which is about 4.5 times higher than inhalation levels. This striking difference in the production of 11-OH-THC is not trivial because 11-OH-THC is an agonist at CB1 receptors, is psychoactive, is believed to pass into the brain more readily than THC (which is consistent with the difference in blood-brain ratios seen for THC vs 11-OH-THC), and is as, or more, potent than THC in its ability to produce behavioural and physiological effects (Grotenhermen, 2003; Lemberger et al., 1972; Perez-Reyes et al., 1972). Recognizing that it is ultimately the activation of CB1 receptors in the brain, which is readily achieved by both THC and possibly more so by 11-OH-THC, the overall impact administration of THC will have on central $\mathrm{CB} 1$ receptor activation must take both THC and 11-OH-THC levels into account. Following injection, extremely high concentrations of 11-OH-THC in the brain will produce different physiological, psychological, and behavioural effects as compared to inhalation. In fact, given the dramatic accumulation of 11-OH-THC in the brain following injection, as well as its significant potency at the $\mathrm{CB} 1$ receptor, it seems reasonable to hypothesize that injection of THC produces a much more robust and sustained activation of brain $\mathrm{CB} 1$ receptors than THC administered via inhalation. As our data indicate that inhalation produces a rapid accumulation of THC in the brain, followed by relatively rapid clearance, this would suggest that the ability of inhaled THC to activate brain CB1 receptors is likely a time-limited effect. This is consistent with our hypothermia 407 data and the relatively rapid peak, and diminution, of psychological effects and intoxication produced by inhaled cannabis or THC. Alternatively, injected THC produces lower initial brain THC concentrations compared to inhaled with levels accumulating over time to reach peak THC levels at 90 min that are comparable to inhaled. However, injection administration also includes the addition of 411 high 11-OH-THC levels, produced through hepatic metabolism, and sequestered into the brain. As 
412 such, injections of THC will potentially produce profoundly different biological effects since the 413 magnitude of CB1 receptor activation (through brain levels of both THC and 11-OH-THC) will be 414 much greater than that following inhaled THC. Given that there is a notable discrepancy between many 415 of the beneficial and adverse impacts of THC that have been documented in rodent studies using 416 injection-based approaches relative to human studies examining cannabis users, one must consider that 417 the injection-based approach for THC has limitations for translational research. As the cellular impacts 418 of CB1 receptor activation will be influenced by the magnitude and duration of its activation, the 419 impacts of accumulating 11-OH-THC in the brain following injections of THC must be considered in 420 future rodent studies.

THC-COOH Levels in Plasma and Brain: Injection administration yielded higher levels of plasma THC-COOH but lower levels of brain THC-COOH compared to inhalation. More specifically, plasma concentrations of THC-COOH were relatively low $(\sim 7 \mathrm{ng} / \mathrm{mL})$ following inhalation and did not differ across timepoints. Whereas, plasma concentrations of THC-COOH were highest at 30, 60, 90 and 240 min compared to $15 \mathrm{~min}$, reaching average peak levels of $\sim 60 \mathrm{ng} / \mathrm{mL}$, about 9 times higher than inhalation levels. Along these lines, previous studies have also shown peak THC-COOH concentrations at later timepoints (60-120 min) following injection in rats (Torrens et al., 2020) and inhalation in humans (Huestis et al., 1992). Further, given that plasma 11-OH-THC concentrations were higher following injection, and 11-OH-THC is the metabolic precursor of THC-COOH, it is not surprising that THC-COOH follows the same pattern. Low levels of $\mathrm{THC}-\mathrm{COOH}$ in the brain are anticipated as it is the primary metabolite for urinary elimination (Huestis, 2005). 
in both brain and blood, indicating that females metabolize THC at a faster rate than males do, which is relatively consistent with previous work (Ruiz et al., 2021; Wiley et al., 2014). In line with accelerated metabolism of THC and increased concentrations of THC metabolites in females, females were found to have lower levels of THC itself relative to males, particularly in the brain. Previous studies have shown no differences between male and female THC blood and brain levels (Javadi-Paydar et al., 440 2017; Nguyen et al., 2020; Ruiz et al., 2021; Wiley et al., 2014). This sex difference in the production 441 of 11-OH-THC, particularly following injections, has relevance for interpreting preclinical studies. For 442 example, many studies report that females are more sensitive to the effects of THC relative to males, 443 particularly in the context of adverse effects of THC. Our data suggests that while females achieve 444 slightly lower brain THC levels than males, they appear to acquire brain 11-OH-THC levels that are 445 essentially double that seen in males following injection of THC. Given the bioactivity and potency of 446 11-OH-THC, this suggests that injection-based studies of THC may suggest sex differences exist in 447 some outcomes, but this effect may be an artifact of the elevated levels of 11-OH-THC produced by 448 injection; an effect which does not occur in the same manner following inhalation where brain 11-OH449 THC levels are quite low and comparable between males and females. These sex differences in THC 450 metabolism may also have implications for human THC consumption, especially when it is consumed 451 via an oral route as entero-hepatic metabolism will impact THC metabolism.

Limitations and Conclusions: Of note, the current studies do not include oral or edible intake of 454 cannabis, another popular form of consumption. This is an area of future work in our group and has 455 recently been successfully executed by others (Kruse et al., 2019). Oral consumption would also result 456 in an increase in 11-OH-THC production due to first pass metabolism; however, given that an 
457 intoxicating dose of oral cannabis in humans produces peak blood levels of THC in the range of 1-5 $458 \mathrm{ng} / \mathrm{m}$ (Vandrey et al., 2017), which is about 1/20-1/100 of the current level produced by injection of 459 THC, one can anticipate that the levels of 11-OH-THC produced would be substantially lower then 460 what we see following injection. Thus, despite the potential similarities in pharmacokinetic trajectories, 461 injections would not be a suitable comparison for oral routes of administration of cannabis or THC. 462 In conclusion, our data demonstrate significant and biologically relevant differences in the 463 pharmacokinetics and accumulation of THC and its metabolites following injection versus inhalation. 464 The current study generally supports previous findings but provides the first direct comparison of both 465 sex and route of administration of THC to reveal an accurate picture of how these variables are 466 impacting THC metabolism and central accumulation. These findings should be considered for 467 translational preclinical studies attempting to model the impacts of cannabis or THC on the brain and 468 behavioural processes. IP injections are the most frequent route of administration for animal models 469 examining the effects of cannabis (THC) and many previous studies claim the translatability and 470 relevance to human consumption by aiming to produce peak plasma THC concentrations that are 471 comparable to concentrations in human cannabis smokers. Utilizing doses that produced comparable 472 peak plasma THC concentrations, our study illustrates robust differences in the pharmacokinetics and 473 central accumulation of THC and its bioactive metabolites when administered via injection versus 474 inhalation. These differences likely underlie the inconsistency of reproducible behavioural findings 475 between THC injections and inhalation, and support the importance of appropriately modeling the route 476 of administration in preclinical studies. This is not uncommon in the drug research field, and in fact 477 studies utilizing injections of ethanol have long been abandoned over the appropriate use of ethanol 478 vapour or drinking as this produces comparable effects to humans, and also allows for the study of 479 volitional administration. Based on the data generated herein, we suggest that researchers conducting 
480 translational work in the realm of THC and cannabis strongly consider utilizing inhalation models, or

481 oral routes of administration, to ensure that any biological effects they see from THC or cannabis

482 extract administration are not artifacts produced by the accumulation of 11-OH-THC in the brain and

483 activating CB1 receptors in a temporal manner that is likely quite distinct from what is occurring with 484 humans during typical cannabis use. 


\section{Acknowledgements}

Thank you to all current and former members of the Hill laboratory for their assistance and expertise. Thank you to Maury Cole and La Jolla Alcohol Research Inc. for his technical assistance with the vapor chambers and Cece Hillard for her input on the manuscript.

\section{Funding}

This study was supported by operating funds from the Canadian Institutes of Health Research (CIHR TCP-431510; MNH) and the Hotchkiss Brain Institute (MNH and SB). CH received salary support from Eyes High Postdoctoral Scholarship and Harley Hotchkiss - Samuel Weiss Postdoctoral Fellowship. SLB received salary support from a Vanier Scholarship from CIHR, GNP received salary support from the Branch Out Neurological Foundation (BONF), RJA received salary support from the Mathison Centre for Mental Health Research \& Education, SHML received salary support from the William H. Davies Medical Research Scholarship. All funding agencies had no influence on the design, execution, or publishing of this work.

\section{Conflict of Interest Statement}

$\mathrm{MNH}$ is a member of the scientific advisory board for Shoppers Drug Mart, Jazz Pharmaceuticals and Lundbeck. 


\section{References}

Abrams, D.I., Vizoso, H.P., Shade, S.B., Jay, C., Kelly, M.E., Benowitz, N.L., 2007. Vaporization as a smokeless cannabis delivery system: A pilot study. Clin. Pharmacol. Ther. 82, 572-578. https://doi.org/10.1038/sj.clpt.6100200

Ashton, C.H., 2001. Pharmacology and effects of cannabis a brief review. Br. J. Psychiatry 178, 101106. https://doi.org/10.1192/bjp.178.2.101

Bidwell, L.C., Ellingson, J.M., Karoly, H.C., Yorkwilliams, S.L., Hitchcock, L.N., Tracy, B.L., Klawitter, J., Sempio, C., Bryan, A.D., Hutchison, K.E., 2020. Association of naturalistic administration of cannabis flower and concentrates with intoxication and impairment. JAMA Psychiatry 9, 1-10. https://doi.org/10.1001/jamapsychiatry.2020.0927

Burstein, S.H., 1999. The cannabinoid acids: Nonpsychoactive derivatives with therapeutic potential. Pharmacol. Ther. 82, 87-96. https://doi.org/10.1016/S0163-7258(98)00069-2

Cuttler, C., Mischley, L.K., Sexton, M., 2016. Sex differences in cannabis use and affects: A crosssectional survey of cannabis users. Cannabis Cannabinoid Res. 1, 166-175. https://doi.org/10.1089/can.2016.0010

ElSohly, M.A., Mehmedic, Z., Foster, S., Gon, C., Chandra, S., Church, J.C., 2016. Changes in cannabis potency over the last 2 decades (1995-2014): Analysis of current data in the United States. Biol. Psychiatry 79, 613-619. https://doi.org/10.1016/j.biopsych.2016.01.004

Freels, T.G., Baxter-Potter, L.N., Lugo, J.M., Glodosky, N.C., Wright, H.R., Baglot, S.L., Petrie, G.N., Yu, Z., Clowers, B.H., Cuttler, C., Fuchs, R.A., Hill, M.N., McLaughlin, R.J., 2020. Vaporized cannabis extracts have reinforcing properties and support conditioned drug-seeking behavior in rats. J. Neurosci. 40, 1897-1908. https://doi.org/10.1523/JNEUROSCI.2416-19.2020

Goodman, S., Wadsworth, E., Leos-Toro, C., Hammond, D., 2020. Prevalence and forms of cannabis 
use in legal vs. illegal recreational cannabis markets. Int. J. Drug Policy 76, 102658.

https://doi.org/10.1016/j.drugpo.2019.102658

Government of Canada, 2019. Cannabis market data. Available from: https:/www.canada.ca/en/healthcanada/services/drugs-medication/cannabis/licensed-producers/market-data/supply-demand.html

Grotenhermen, F., 2003. Pharmacokinetics and pharmacodynamics of cannabinoids. Clin.

Pharmacokinet. 42, 327-360. https://doi.org/10.2165/00003088-200342040-00003

Hartman, R.L., Brown, T.L., Milavetz, G., Spurgin, A., Gorelick, D.A., Gaffney, G., Huestis, M.A., 2015. Controlled cannabis vaporizer administration: Blood and plasma cannabinoids with and without alcohol. Clin. Chem. 61, 850-869. https://doi.org/10.1373/clinchem.2015.238287

Hložek, T., Uttl, L., Kadeřábek, L., Balíková, M., Lhotková, E., Horsley, R.R., Nováková, P., Šíchová, K., Štefková, K., Tylš, F., Kuchař, M., Páleníček, T., 2017. Pharmacokinetic and behavioural profile of $\mathrm{THC}, \mathrm{CBD}$, and $\mathrm{THC}+\mathrm{CBD}$ combination after pulmonary, oral, and subcutaneous administration in rats and confirmation of conversion in vivo of CBD to THC. Eur. Neuropsychopharmacol. 27, 1223-1237. https://doi.org/10.1016/j.euroneuro.2017.10.037 Huestis, M.A., 2007. Human cannabinoid pharmacokinetics. Chem. Biodivers. 4, 1770-1804. https://doi.org/10.1002/cbdv.200790152

Huestis, M.A., 2005. Pharmacokinetics and metabolism of the plant cannabinoids, $\Delta 9$ tetrahydrocannabinol, cannabidiol and cannabinol. Handb. Exp. Pharmacol. 168, 657-690.

Huestis, M.A., Cone, E.J., 2004. Relationship of $\Delta$ 9-tetrahydrocannabinol concentrations in oral fluid and plasma after controlled administration of smoked cannabis. J. Anal. Toxicol. 28, 394-399. https://doi.org/10.1093/jat/28.6.394

Huestis, M.A., Henningfield, J.E., Cone, E.J., 1992. Blood cannabinoids. ii. models for the prediction of time of marijuana exposure from plasma concentrations of $\Delta 9$-tetrahydrocannabinol (thc)and 
11-nor-9-carboxy- $\Delta$ 9- tetrahydrocannabinol (thccooh). J. Anal. Toxicol. 16, 283-290. https://doi.org/10.1093/jat/16.5.283

Javadi-Paydar, M., Nguyen, J., Kerr, T., Grant, Y., Vandewater, S., Cole, M., Taffe, M., 2017. Effects of $\triangle 9$-THC and cannabidiol vapor inhalation in male and female rats. Psychopharmacology (Berl). 235, 2541-2557. https://doi.org/10.1101/128173

Kirkham, T.C., Williams, C.M., 2005. Endogenous cannabinoids and appetite. Nutr. Res. Rev. 14, 6586. https://doi.org/10.1079/095442201108729141

Kruse, L.C., Cao, J.K., Viray, K., Stella, N., Clark, J.J., 2019. Voluntary oral consumption of $\Delta 9$ tetrahydrocannabinol by adolescent rats impairs reward-predictive cue behaviors in adulthood. Neuropsychopharmacology 44, 1406-1414. https://doi.org/10.1038/s41386-019-0387-7

Kulkarni, A.D., Patel, H.M., Surana, S.J., Belgamwar, V.S., Pardeshi, C. V., 2016. Brain-blood ratio: Implications in brain drug delivery. Expert Opin. Drug Deliv. 13, 85-92. https://doi.org/10.1517/17425247.2016.1092519

Lemberger, L., Crabtree, R.E., Rowe, H.M., 1972. 11-hydroxy- $\Delta$ 9-tetrahydrocannabinol: pharmacology, disposition, and metabolism of a major metabolite of marihuana in man. Science. 177, 62-64. https://doi.org/10.1126/science.177.4043.62

Lukas, G., Brindle, S.D., Greengard, P., 1971. The route of absorption of intraperitoneally administered compounds. J. Pharmacol. Exp. Ther. 178, 562-566.

Manwell, L.A., Ford, B., Matthews, B.A., Heipel, H., Mallet, P.E., 2014. A vapourized $\delta 9-$ tetrahydrocannabinol ( $89-\mathrm{THC})$ delivery system part II: Comparison of behavioural effects of pulmonary versus parenteral cannabinoid exposure in rodents. J. Pharmacol. Toxicol. Methods 70, 112-119. https://doi.org/10.1016/j.vascn.2014.06.004

Newmeyer, M.N., Swortwood, M.J., Barnes, A.J., Abulseoud, O.A., Scheidweiler, K.B., Huestis, M.A., 
2016. Free and glucuronide whole blood cannabinoids' pharmacokinetics after controlled smoked, vaporized, and oral cannabis administration in frequent and occasional cannabis users:

Identification of recent cannabis intake. Clin. Chem. 62, 1579-1592.

https://doi.org/10.1373/clinchem.2016.263475

Nguyen, J.D., Aarde, S.M., Vandewater, S.A., Grant, Y., Stouffer, D.G., Parsons, L.H., Cole, M.,

Taffe, M.A., 2016. Inhaled delivery of $\Delta$ 9-tetrahydrocannabinol (THC) to rats by e-cigarette vapor technology. Neuropharmacology 109, 112-120. https://doi.org/10.1016/j.neuropharm.2016.05.021

Nguyen, J.D., Creehan, K.M., Kerr, T.M., Taffe, M.A., 2020. Lasting effects of repeated $\Delta$ 9-

tetrahydrocannabinol vapour inhalation during adolescence in male and female rats. Br. J.

Pharmacol. 177, 188-203. https://doi.org/10.1111/bph.14856

Perez-Reyes, M., Timmons, M.C., Lipton, M.A., Davis, K.H., Wall, M.E., 1972. Intravenous injection in man of $\$ \backslash$ delta $\wedge\{9\} \$$ tetrahydrocannabinol and 11-OH-\$delta $\wedge\{9\} \$$-tetrahydrocannabinol.

Science. 177, 633-635.

Ruiz, C.M. et al., 2021. Pharmacokinetic, behavioral, and brain activity effects of ?9-

tetrahydrocannabinol in adolescent male and female rats. Neuropsychopharmacology 46, 959969. https://doi.org/10.1038/s41386-020-00839-w

Schwope, D.M., Karschner, E.L., Gorelick, D.A., Huestis, M.A., 2011. Identification of recent cannabis use: whole-blood and plasma free glucuronidated cannabinoid pharmacokinetics following controlled smoked cannabis adminsitration. Clin. Chem. 57, 1406-1414.

Sharma, P., Murthy, P., Bharath, M.M.S., 2012. Chemistry, metabolism, and toxicology of cannabis:

Clinical implications. Iran. J. Psychiatry 7, 149-156.

Spindle, T.R., Cone, E.J., Schlienz, N.J., Mitchell, J.M., Bigelow, G.E., Flegel, R., Hayes, E., Vandrey, R., 2019. Acute pharmacokinetic profile of smoked and vaporized cannabis in human blood and 
oral fluid. J. Anal. Toxicol. 43, 233-258. https://doi.org/10.1093/jat/bky104

Spindle, T.R., Cone, E.J., Schlienz, N.J., Mitchell, J.M., Bigelow, G.E., Flegel, R., Hayes, E., Vandrey, R., 2018. Acute effects of smoked and vaporized cannabis in healthy adults who infrequently use cannabis: A crossover trial. JAMA Netw. Open 1, e184841.

https://doi.org/10.1001/jamanetworkopen.2018.4841

Taffe, M.A., Creehan, K.M., Vandewater, S.A., Kerr, T.M., Cole, M., 2020. Effects of $\Delta 9$ tetrahydrocannabinol (THC) vapor inhalation in sprague-dawley and wistar rats. Exp. Clin. Psychopharmacol. Advance online publication. https://doi.org/10.1037/1064-1297.11.3.c2

Torrens, A., Vozella, V., Huff, H., Mcneil, B., Das, A., Piomelli, D., 2020. Comparative pharmacokinetics of $\Delta 9$-tetrahydrocannabinol in adolescent and adult male mice. J. Pharmacol. Exp. Ther. https://doi.org/10.1124/jpet.120.265892

Turner, P. V., Brabb, T., Pekow, C., Vasbinder, M.A., 2011. Administration of substances to laboratory animals: Routes of administration and factors to consider. J. Am. Assoc. Lab. Anim. Sci. 50, 600613.

Vandrey, R., Herrmann, E.S., Mitchell, J.M., Bigelow, G.E., Flegel, R., LoDico, C., Cone, E.J., 2017. Pharmacokinetic profile of oral cannabis in humans: Blood and oral fluid disposition and relation to pharmacodynamic outcomes. J. Anal. Toxicol. 41, 83-99. https://doi.org/10.1093/jat/bkx012

Watanabe, K., Tanaka, T., Yamamoto, I., Yoshimura, H., 1988. Brain microsomal oxidation of $\Delta 8-$ and $\Delta$ 9-tetrahydrocannabinol. Biochem. Biophys. Res. Commun. 157, 75-80. https://doi.org/10.1016/S0006-291X(88)80013-5

Wiley, J.L., Burston, J.J., 2014. Sex differences in $\delta 9$-tetrahydrocannabinol metabolism and in vivo pharmacology following acute and repeated dosing in adolescent rats. Neurosci. Lett. 576, 51-55. https://doi.org/10.1016/j.neulet.2014.05.057 
Table 1: Multiple Reaction Monitoring (MRM) Transitions and Collision Energies (CE) of different compounds/standards

\begin{tabular}{|c|c|c|c|c|}
\hline Compounds/Standards & $\begin{array}{c}\text { Q1 } \\
\text { (Da) }\end{array}$ & $\begin{array}{c}\text { Q3 } \\
\text { (Da) }\end{array}$ & $\begin{array}{c}\text { Retention } \\
\text { Time (min) }\end{array}$ & $\begin{array}{c}\text { CE } \\
\text { (volt) }\end{array}$ \\
\hline 11-OH-THC-1 & 331 & 313 & 2 & 27 \\
\hline 11-OH-THC-2 & 331 & 193 & 2 & 27 \\
\hline THC-COOH-1 & 345 & 327 & 2 & 24 \\
\hline THC-COOH-2 & 345 & 299 & 2 & 24 \\
\hline THC-COOH-d3-1 & 348 & 330 & 2 & 24 \\
\hline THC-COOH-d3-2 & 348 & 302 & 2 & 24 \\
\hline THC-1 & 315 & 193 & 2.7 & 30 \\
\hline THC-2 & 315 & 259 & 2.7 & 30 \\
\hline THC-d3-1 & 318 & 196 & 2.7 & 30 \\
\hline THC-d3-2 & 318 & 262 & 2.7 & 30 \\
\hline
\end{tabular}




\section{A. Illustration of passive vapour delivery system}

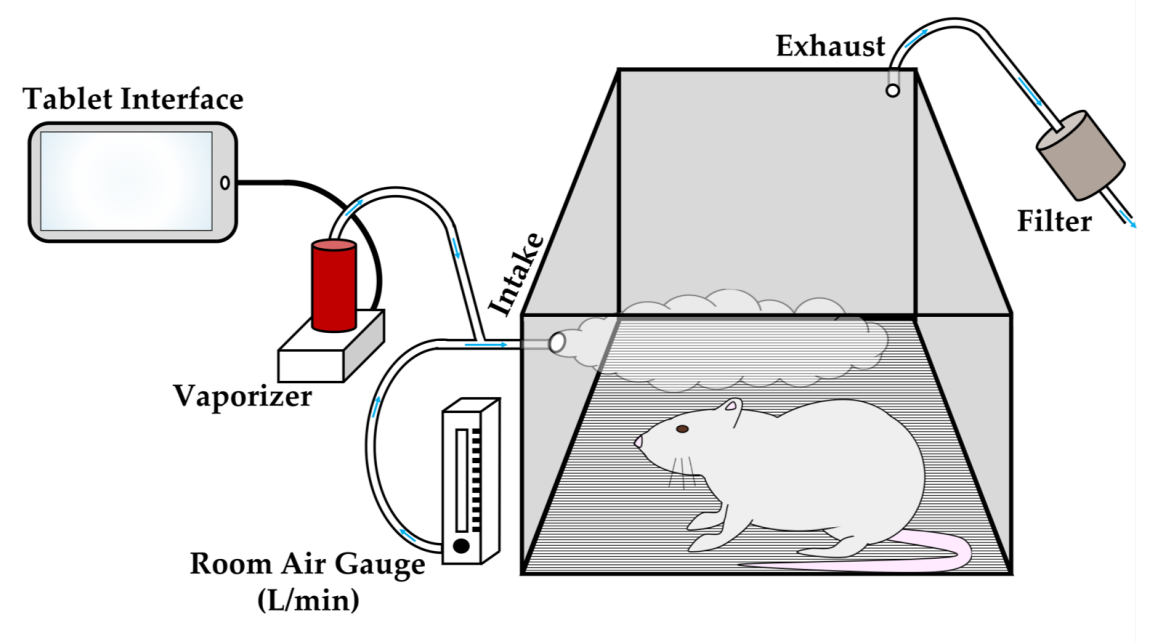

\section{B. Image of passive exposure}

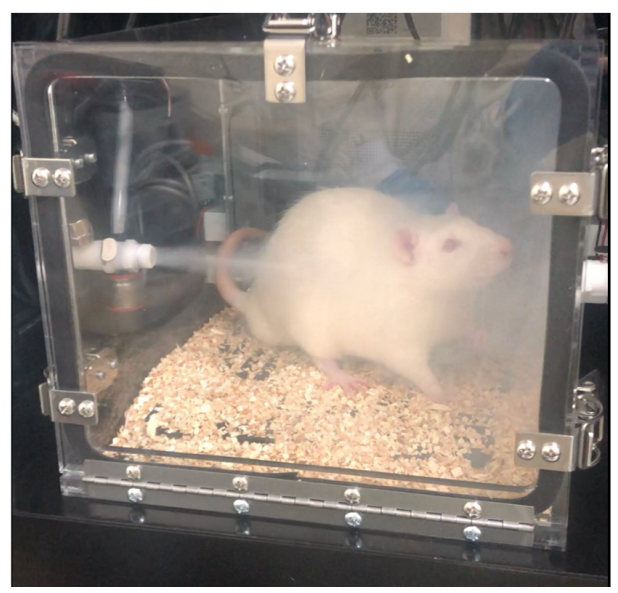

\section{Figure 1: Vapor Delivery System.}

A. Illustration of passive vapour delivery system: Schematic of vapour apparatus components with direction of airflow (adapted from Freels et al. 2020). Briefly, the vapour inhalation system uses machinery similar to electronic cigarettes to deliver distinct "puffs" of vapour within airtight chambers. A vacuum compressor pulls ambient room air through an intake valve at a constant rate of $1 \mathrm{~L}$ per minute. At set intervals THC distillate is vaporized combining with the constant ambient air flow for delivery into the chamber. Air (and vapour) are evacuated through the back of the chamber via the vacuum compressor, filtered and ventilated out of the building. B. Image of passive exposure: Picture of a male SD rat within the vapour apparatus. 


\section{A. Male Temperature}

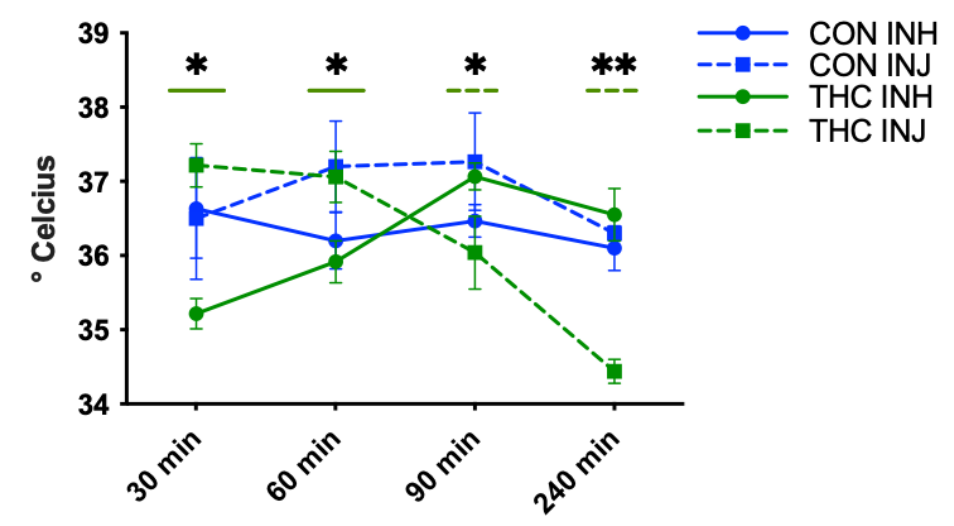

\section{B. Female Temperature}

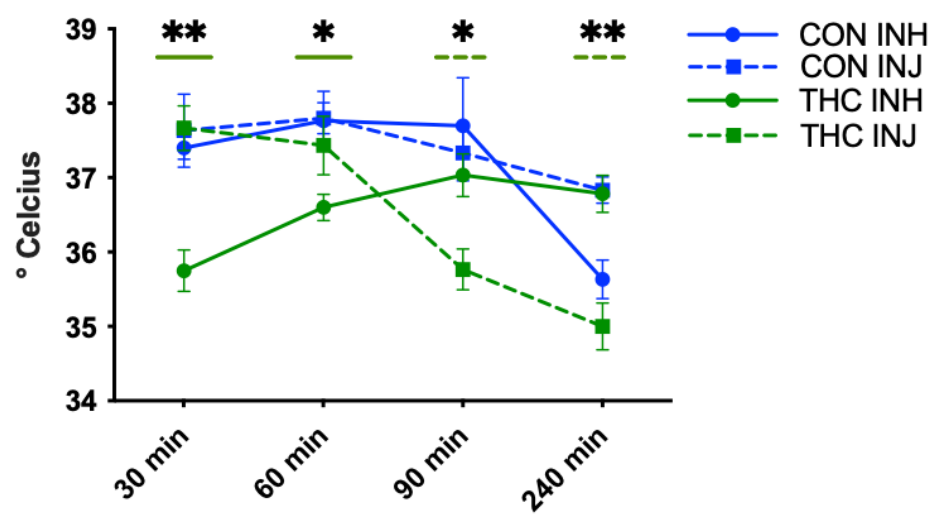

\section{Figure 2: Body Temperature}

A. Male temperature: Data are presented as mean \pm SEM; $n=5-6$ for each group. Presence of $(*)$ with a solid and dashed green line indicates that THC INH and THC INJ respectively differ from one or more other groups. THC INH resulted in immediate hypothermia at $30 \mathrm{~min}(\mathrm{THC} \mathrm{INH}<$ all groups at $* \mathrm{p}<0.05)$ and at $60 \mathrm{~min}(\mathrm{THC} \mathrm{INH}<\mathrm{CON}$ INJ and THC INJ at *p<0.05), whereas THC INJ resulted in delayed hypothermia at 90 min (THC INJ $<$ CON INJ and THC INH at $* \mathrm{p}<0.05)$ and at $240 \mathrm{~min}(\mathrm{THC} \mathrm{INJ}<$ all groups at $* * \mathrm{p}<0.01)$. B. Female temperature: Data are presented as mean \pm SEM; $n=5-6$ for each group. Presence of $\left({ }^{*}\right)$ with a solid and dashed green line indicates that THC INH and THC INJ respectively differ from one or more other groups. THC INH resulted in immediate hypothermia at $30 \mathrm{~min}$ (THC INH $<$ all groups at $* * \mathrm{p}<0.01$ ) and at $60 \mathrm{~min}(\mathrm{THC} \mathrm{INH}<$ all groups at $* \mathrm{p}<0.05$ ), whereas THC INJ resulted in delayed hypothermia at $90 \mathrm{~min}$ (THC INJ < all groups at * $<<0.05$ ) and at $240 \mathrm{~min}$ (THC INJ $<$ CON INJ and THC INH at **p $<0.01)$. 


\section{A. THC Chromatogram}

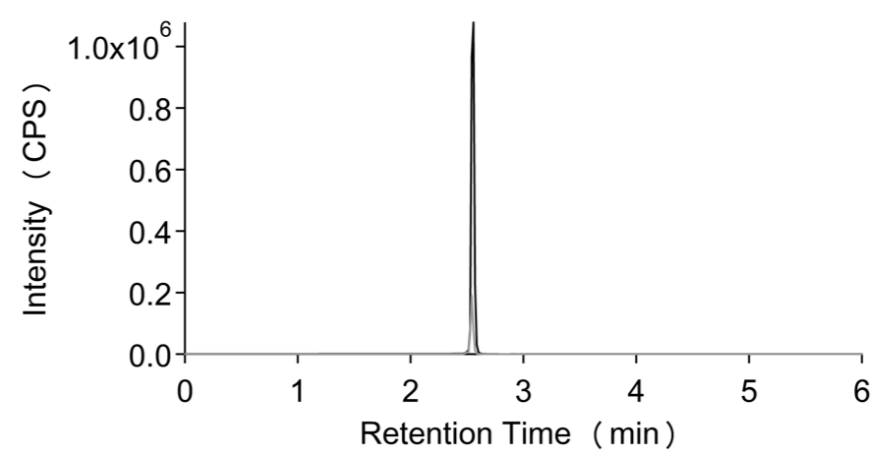

\section{B. THC Blood Levels}

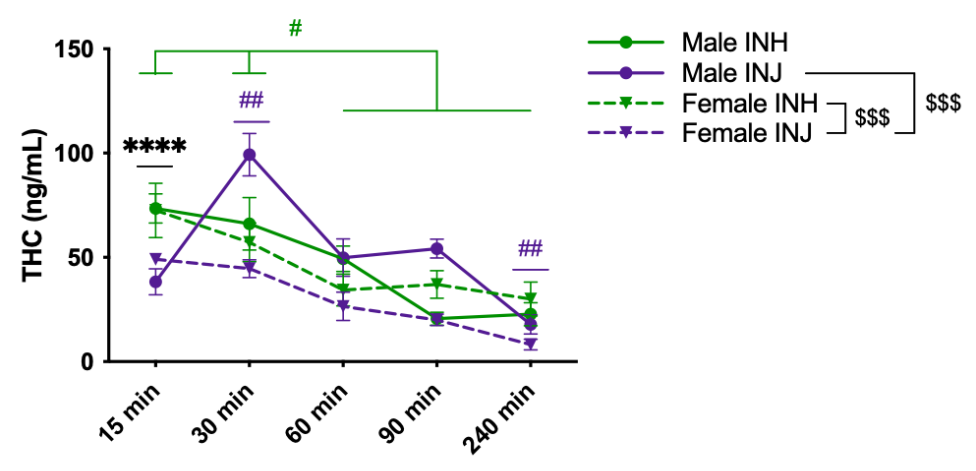

\section{THC Brain Levels}

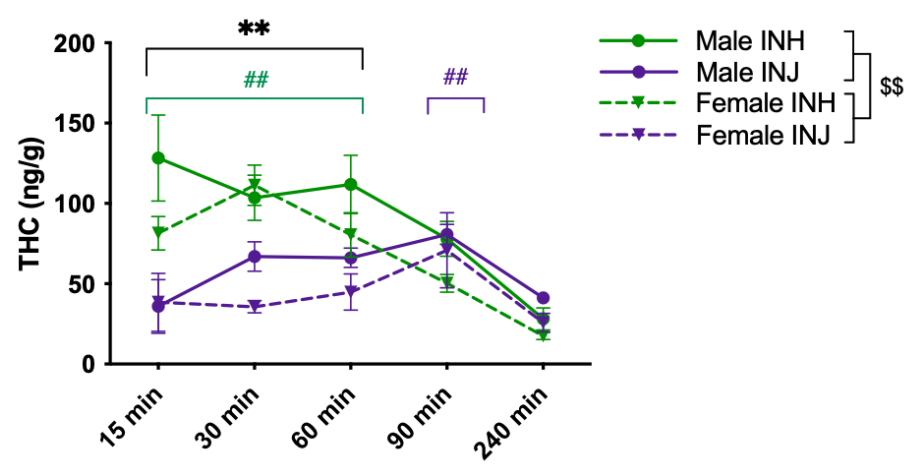

Figure 3: THC Chromatogram and Levels in Blood and Brain.

A. LC-MS Chromatogram: THC (black line) and THC-d3 (grey line) overlapping peaks at $2.7 \mathrm{~min}$. B. Blood levels: Data are presented as mean $\pm \mathrm{SEM} ; \mathrm{n}=5-6$ for each group. Presence of $(*)$ indicates an administration difference with $\mathrm{INH}>\mathrm{INJ}$ at $15 \mathrm{~min}$ at $* * * * \mathrm{p}<0.0001$. Presence of $\left(^{\#}\right)$ indicates a timepoint difference with green and purple lines indicating an INH and INJ difference respectively; specifically, INH $15>$ all timepoints and INH 30> INH 60/90/240 at ${ }^{\#} \mathrm{p}<0.05$, whereas INJ $30>$ all timepoints and INJ $240<$ all timepoints at ${ }^{\# \#} \mathrm{p}<0.01$. Presence of $\left({ }^{\$}\right)$ indicates a sex difference; specifically, female INH $>$ female INJ at ${ }^{\$ \$} p<0.001$ and male INJ $>$ female INJ at ${ }^{\$ \$} p<0.001$ ). C. Brain levels: Data are presented as mean $\pm \mathrm{SEM} ; \mathrm{n}=5-6$ for each group. Presence of $(*)$ indicates an administration difference with INH $>$ INJ at 15,30 , and $60 \mathrm{~min}$ at $* * p<0.01$. Presence of $\left(^{\#}\right)$ indicates a timepoint difference with green and purple lines indicating an INH and INJ difference respectively; specifically, INH 15, 30, and $60>$ INH 90 and 240 at ${ }^{\#} \mathrm{p}<0.01$, whereas INJ $90>$ INJ 15 and 240 at ${ }^{\# \#} \mathrm{p}<0.01$. Presence of $\left({ }^{\$}\right)$ indicates a sex difference with males $>$ females at ${ }^{\$ \$} \mathrm{p}<0.01$ 


\section{A. 11-OH-THC Chromatogram}

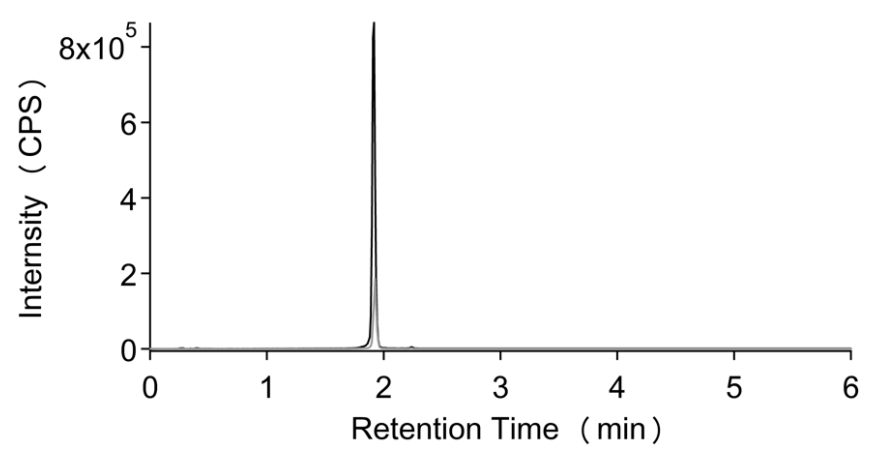

\section{B. 11-OH-THC Blood Levels}

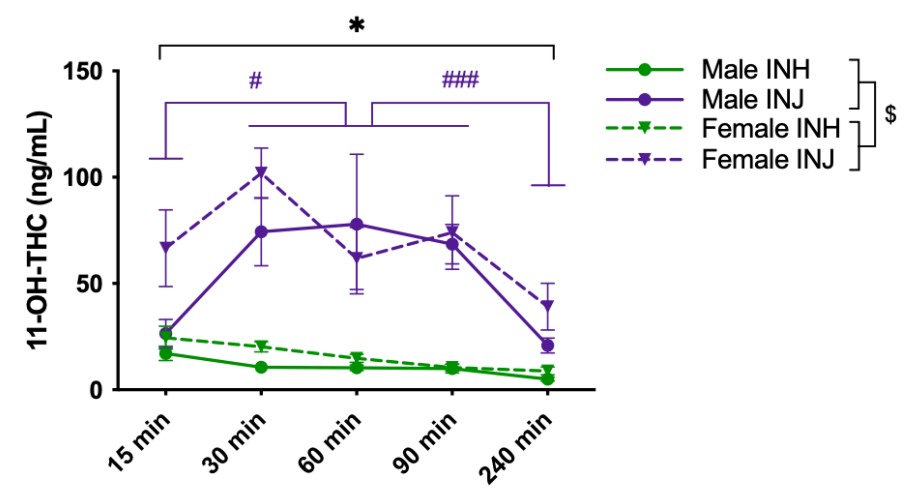

\section{11-OH-THC Brain Levels}

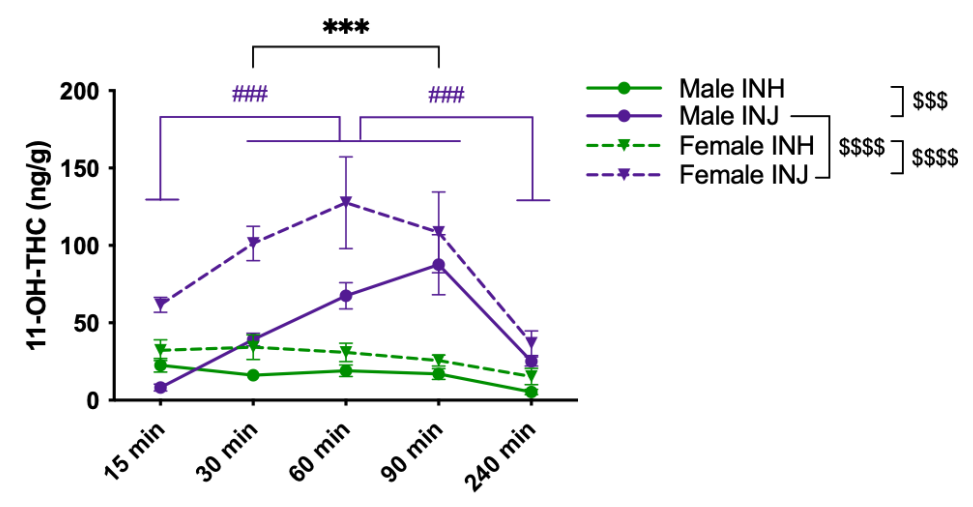

\section{Figure 4: 11-OH-THC Chromatogram and Levels in Blood and Brain.}

A. LC-MS Chromatogram: 11-OH-THC (black line) and 11-OH-THC-d3 (grey line) peaks at $2.0 \mathrm{~min}$. B. Blood levels: Data are presented as mean \pm SEM; $n=5-6$ for each group. Presence of $(*)$ indicates an administration difference with $\mathrm{INH}<\mathrm{INJ}$ at all timepoints at ${ }^{*} \mathrm{p}<0.05$. Presence of $\left({ }^{*}\right)$ indicates a timepoint difference with purple lines indicating an INJ difference where INJ $15<$ INJ 30, 60, and 90 at ${ }^{\#} p<0.05$ and INJ $240<$ INJ 30, 60, and 90 at ${ }^{\# \# \#} \mathrm{p}<0.001$. Presence of $\left({ }^{\$}\right)$ indicates a sex difference; specifically, females $>$ males at ${ }^{\$} \mathrm{p}<0.05$. C. Brain levels: Data are presented as mean \pm SEM; $n=5-6$ for each group. Presence of $\left(^{*}\right)$ indicates an administration difference with INH $<$ INJ at 30, 60, and $90 \mathrm{~min}$ at $* * * \mathrm{p}<0.001$. Presence of $(\#)$ indicates a timepoint difference with purple lines indicating an INJ difference where INJ 15 and $240<$ INJ 30, 60, and 90 at ${ }^{\# \#} p<0.001$. Presence of $\left({ }^{\$}\right)$ indicates a sex difference; specifically, male INH $<$ male INJ at ${ }^{\$ \$} \mathrm{p}<0.001$, female INH $<$ female INJ at ${ }^{\$ \$ \$} \mathrm{p}<0.0001$, and male INJ $<$ female INJ at ${ }^{\$ \$ \$} \mathrm{p}<0.0001$. 


\section{A. THC-COOH Chromatogram}

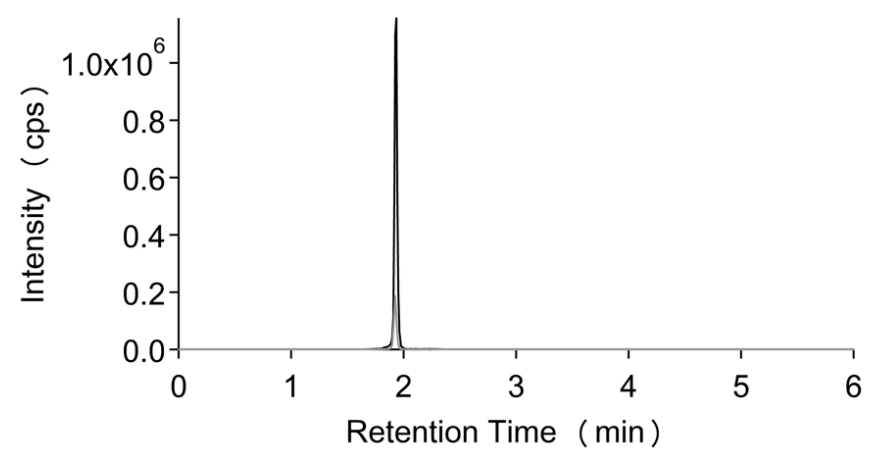

\section{B. THC-COOH Blood Levels}

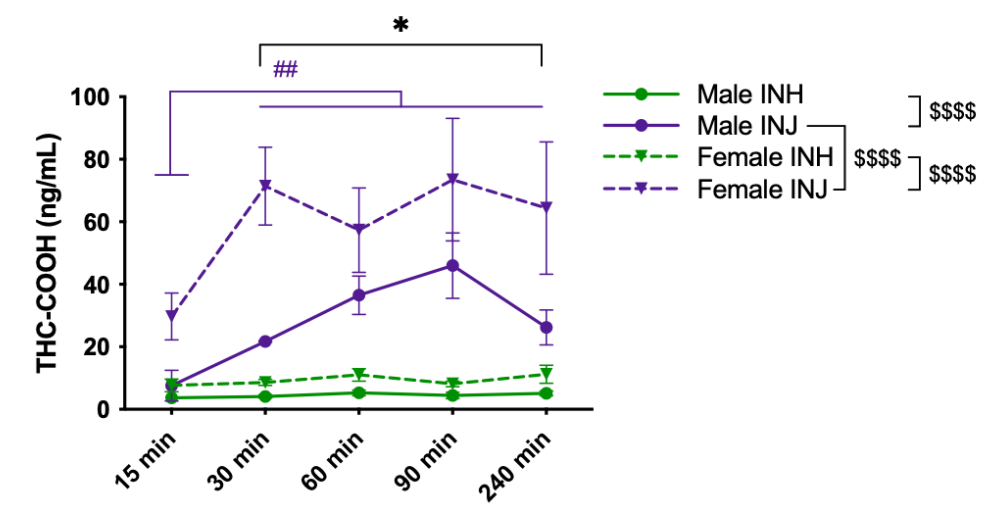

\section{THC-COOH Brain Levels}

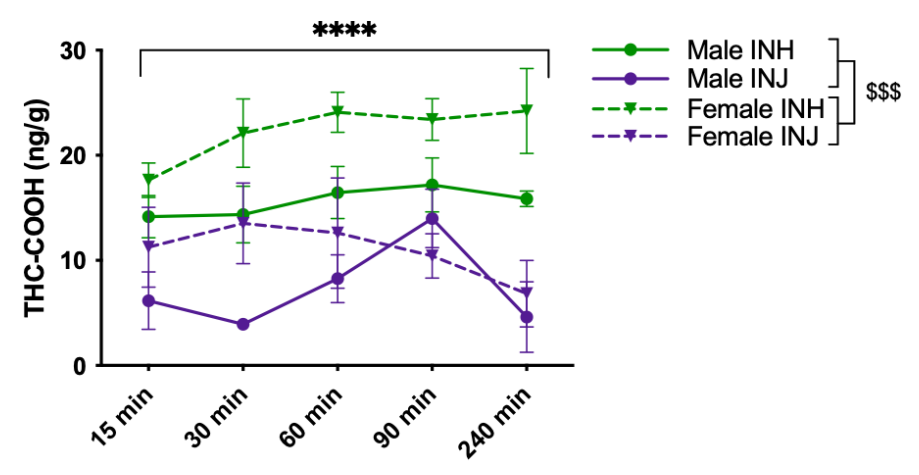

\section{Figure 5: THC-COOH Chromatogram and Levels in Blood and Brain.}

A. LC-MS Chromatogram: THC-COOH (black line) and THC-COOH-d3 (grey line) peaks at $2.0 \mathrm{~min}$. B. Blood levels: Data are presented as mean $\pm \mathrm{SEM} ; \mathrm{n}=5-6$ for each group. Presence of $(*)$ indicates an administration difference with INH $<\mathrm{INJ}$ at 30,60, 90 and $240 \mathrm{~min}$ at $* \mathrm{p}<0.05$. Presence of $\left(^{*}\right)$ indicates a timepoint difference with purple lines indicating an INJ difference where INJ $15<$ all timepoints at ${ }^{\#} p<0.01$. Presence of $\left({ }^{\$}\right)$ indicates a sex difference; specifically, male INH $<$ male INJ and female INH $<$ female INJ at ${ }^{\$ \$ \$}$ p $<0.0001$, and male INJ $<$ female $\mathrm{INJ}$ at ${ }^{\$ \$ \$} \mathrm{p}<0.0001$. C. Brain levels: Data are presented as mean $\pm \mathrm{SEM} ; \mathrm{n}=5-6$ for each group. Presence of $(*)$ indicates an administration difference with INH $>$ INJ at $* * * * p<0.0001$. Presence of $\left({ }^{\$}\right)$ indicates a sex difference with males $<$ females at ${ }^{\$ \$} \mathrm{p}<0.001$. 